\title{
On a modification of Olver's method: a special case
}

\author{
Chelo Ferreira $^{1}$, José L. López ${ }^{2}$ and Ester Pérez Sinusía ${ }^{1}$ \\ ${ }^{1}$ Dpto. de Matemática Aplicada, IUMA, Universidad de Zaragoza \\ e-mail: cferrei@unizar.es, ester.perez@unizar.es \\ ${ }^{2}$ Dpto. de Ingeniería Matemática e Informática, Universidad Pública de Navarra and INAMAT, Navarra \\ e-mail: jl.lopez@unavarra.es
}

\begin{abstract}
We consider the asymptotic method designed by F. Olver [Olver, 1974] for linear differential equations of the second order containing a large (asymptotic) parameter $\Lambda: x^{m} y^{\prime \prime}-\Lambda^{2} y=$ $g(x) y$, with $m \in \mathbb{Z}$ and $g$ continuous. Olver studies in detail the cases $m \neq 2$, especially the cases $m=0, \pm 1$, giving the Poincaré-type asymptotic expansions of two independent solutions of the equation. The case $m=2$ is different, as the behavior of the solutions for large $\Lambda$ is not of exponential type, but of power type. In this case, Olver's theory does not give many details. We consider here the special case $m=2$. We propose two different techniques to handle the problem: (i) a modification of Olver's method that replaces the role of the exponential approximations by power approximations and (ii) the transformation of the differential problem into a fixed point problem from which we construct an asymptotic sequence of functions that converges to the unique solution of the problem. Moreover, we show that this second technique may also be applied to nonlinear differential equations with a large parameter.
\end{abstract}

2010 AMS Mathematics Subject Classification: 34A12; 41A58; 41A60; 34B27.

Keywords \& Phrases: Second order differential equations. Asymptotic expansions. Green's functions. Banach's fixed point theorem.

\section{Introduction}

The most famous asymptotic method for second order linear differential equations containing a large parameter is, no doubt, Olver's method. In [5, Chaps. 10, 11, 12], Olver considers the differential equation

$$
u^{\prime \prime}-\frac{\tilde{\Lambda}^{2}}{z^{m}} u=h(z) u, \quad \tilde{\Lambda} \rightarrow \infty
$$

with $m=0,-1,1, \tilde{\Lambda}$ is a complex parameter, $z$ is a complex variable and $h$ is an analytic function in a certain region of the complex plane, although Olver also considers cases in which $h(z)$ could have a double pole. Correspondingly to these three different $m$-cases, Olver divides 
the study of (1) in three canonical cases, say I, II and III, analyzed in Chapters 10, 11 and 12 respectively. In Case I, Olver completes the theory developed in the well-known Liouville-Green approximation, giving a rigorous meaning to the approximation and providing error bounds for the expansions of solutions of (1) for $m=0$. In Cases II and III, Olver extends the theory introduced in Case I considering, respectively, the case $m=-1$ (differential equations with a turning point) and the case $m=1$ (differential equations with a regular singular point).

In [5, Chap. 12, Sec. 14] we can also find indications about the generalization of the study of the asymptotics of the solutions of (1) for general $m \in \mathbb{Z}$, except $m=2$. In summary, we have that for any $m \in \mathbb{Z} \backslash\{2\}$, two independent solutions of (1) have the form

$$
u(z)=P_{m}(z)\left[\sum_{k=0}^{n-1} \frac{A_{k}(z)}{\tilde{\Lambda}^{2 k}}+R_{m, n}(z)\right]+\frac{1}{\tilde{\Lambda}^{2}} P_{m}^{\prime}(z)\left[\sum_{k=0}^{n-1} \frac{B_{k}(z)}{\tilde{\Lambda}^{2 k}}+\bar{R}_{m, n}(z)\right]
$$

where $R_{m, n}(z), \bar{R}_{m, n}(z)=\mathcal{O}\left(\tilde{\Lambda}^{-2 n}\right)$ uniformly for $z$ in a certain region in the complex plane. In this formula, $P_{m}(z)$ is one of the two following basic solutions of (1), that is, independent solutions of (1) for $h=0$ :

$$
P_{m}(z):=\left\{\begin{array}{l}
\sqrt{z} I_{\hat{m}}\left(2 \hat{m} \tilde{\Lambda} z^{1 /(2 \hat{m})}\right), \\
\sqrt{z} K_{\hat{m}}\left(2 \hat{m} \tilde{\Lambda} z^{1 /(2 \hat{m})}\right),
\end{array} \quad \hat{m}:=\frac{1}{2-m} .\right.
$$

In this formula and in the remaining of the paper, the symbols $I_{\nu}(z)$ and $K_{\nu}(z)$ denote the principal values of the modified Bessel functions. For example, for $m=0,1,3,4,5, \ldots$, the coefficients $A_{k}$ and $B_{k}$ are given by the following system of recurrences: $A_{0}(z)=1$ and

$$
\begin{array}{rlrl}
B_{n}(z) & =\frac{z^{m / 2}}{2} \int z^{m / 2}\left[h(z) A_{n}(z)-A_{n}^{\prime \prime}(z)\right] d z, & \\
A_{n+1}(z) & =-\frac{1}{2} B_{n}^{\prime}(z)+\frac{1}{2} \int h(z) B_{n}(z) d z, & &
\end{array}
$$

Both families of coefficients $A_{n}$ and $B_{n}$ are analytic at $z=0$ when $h(z)$ is also analytic there. Olver's important contribution is the proof of the asymptotic character of the two expansions (2)-(3) and the derivation of error bounds for the remainder $R_{m, n}(z)$.

For large $\tilde{\Lambda}$ and fixed $z$, both solutions have an asymptotic behavior of exponential type $[6$, Sec. 10.30(ii)]

$$
\begin{aligned}
\sqrt{z} I_{\hat{m}}\left(2 \hat{m} \tilde{\Lambda} z^{1 /(2 \hat{m})}\right) & =\mathcal{O}\left(\frac{z^{m / 4}}{\sqrt{\tilde{\Lambda}}} e^{2\left|\hat{m} \Re\left(\tilde{\Lambda} z^{1 /(2 \hat{m})}\right)\right|}\right), \\
\sqrt{z} K_{\hat{m}}\left(2 \hat{m} \tilde{\Lambda} z^{1 /(2 \hat{m})}\right) & =\mathcal{O}\left(\frac{z^{m / 4}}{\sqrt{\tilde{\Lambda}}} e^{-2 \hat{m} \Re\left(\tilde{\Lambda} z^{1 /(2 \hat{m})}\right)}\right),
\end{aligned}
$$

both valid in the sector $\left|\operatorname{Arg}\left(\tilde{\Lambda} z^{1 /(2 \hat{m})}\right)\right|<3 \pi / 2$. Therefore, for any $m \neq 2$, two independent solutions of (1) have an exponential asymptotic behavior for large $\tilde{\Lambda}$ and fixed $z$. The above approximations obviously fail for $m=2$. This case is considered by Olver in [5, Chap. 6, Sec. 5.3], where he gives the first order asymptotic approximation (WKB approximation) for two independent solutions of (1). Also, in [5, Chap. 10, Sec. 4.1], Olver gives some indications 
about the derivation of a complete asymptotic expansion in terms of the expansion given for the case $m=0$, although details are not given there.

The purpose of this paper is to analyze the asymptotic behavior of the solutions of the equation $u^{\prime \prime}-\tilde{\Lambda}^{2} z^{-2} u=h(z) u$ in detail. To this end, in the next section we introduce an appropriate change of the unknown in the differential equation. In Section 3, we use a fixed point theorem and the Green function of an auxiliary initial value problem to derive an asymptotic as well as convergent expansion of a couple of independent solutions of the equation in terms of iterated integrals of $h(z)$; this technique is based on our previous investigations [4]. In Section 4 , we generalize this technique to nonlinear problems, where we obtain an asymptotic expansion of an initial value problem for a nonlinear equation. In Section 5, we use Olver's techniques to obtain asymptotic expansions, of Poincaré-type, of two independent solutions of the equation, different from those obtained in Section 3. Section 6 contains and example and some numerical experiments and Section 7 a few remarks and conclusions.

\section{Preliminaries}

Consider the differential equation (1) with $m=2$. For later convenience, we define the function $g(z):=z h(z)$ and a new large parameter

$$
\Lambda:=\frac{1+\sqrt{4 \tilde{\Lambda}^{2}+1}}{2} .
$$

In terms of this parameter and the new function $g(z)$, equation (1) with $m=2$ reads

$$
z^{2} u^{\prime \prime}(z)-\Lambda(\Lambda-1) u(z)=z g(z) u(z) .
$$

Because this equation is invariant under the transformation $\Lambda \rightarrow 1-\Lambda$, in the remaining of the paper, and without loss of generality, we consider $\Re \Lambda \geq 1 / 2,2 \Lambda \neq 1$. As we mentioned in the introduction, the general formula (2) is not directly applicable to this equation; for $m=2$, the index $\hat{m}$ of the basic Bessel functions approximants in (3) becomes infinite, the asymptotic behavior of the solutions of (5) is not exponential in $\Lambda$. On the other hand, as it is explained in [3], when we consider this equation with an initial condition at the point $z=0$, a fixed point technique does not work either: the exponent $m=2$ in the coefficient $z^{2}$ of $u^{\prime \prime}$ makes the iterated integrals related to the fixed point iterations divergent at $z=0$.

Both problems may be overcome by means of an appropriate change of unknown $u \rightarrow y$ that modifies the exponent $m=2$. In order to perform the appropriate change of unknown, we consider here the Frobenius theory. When the function $g(z)$ is analytic at $z=0$, the exponents of the Frobenius solutions of the differential equation (5) at the regular singular point $z=0$ are $\mu_{1}=\Lambda$ and $\mu_{2}=1-\Lambda$. Therefore, two independent solutions of this equation behave, at $z=0$, as $z^{\Lambda}$ and $z^{1-\Lambda}$ respectively. This fact suggests the following change of unknown: $u \rightarrow y:=z^{-\Lambda} u$. The new unknown $y$ satisfies the differential equation

$$
z y^{\prime \prime}(z)+2 \Lambda y^{\prime}(z)=g(z) y(z) .
$$

When $g(z)$ is an analytic function at $z=0$ we know, from Frobenius theory, that this equation has two independent solutions that behave, at $z=0$, as 1 and $z^{1-2 \Lambda}$ respectively. Therefore, in the linear two-dimensional space of solutions of this equation, only one ray of solutions is 
bounded at $z=0$. These facts determine the kind of possible well-posed problems for this equation. A well-posed initial value problem for the differential equation (6) with initial datum given at $z=0$ is

$$
\left\{\begin{array}{l}
z y^{\prime \prime}(z)+2 \Lambda y^{\prime}(z)=g(z) y(z) \quad \text { in } \mathcal{D} \\
y(0)=\bar{y}_{0}
\end{array}\right.
$$

where $\bar{y}_{0}$ is any complex parameter, $\bar{y}_{0}=\mathcal{O}(1)$ as $\Lambda \rightarrow \infty$, and $\mathcal{D}$ is a star-like domain (bounded or unbounded) in the complex plane centered at $z=0$. In the next section, we will show that this problem has a unique solution and we will obtain an asymptotic approximation of the unique solution of this problem. In order to derive an asymptotic expansion of a second independent solution of $(6)$, we must consider an initial value problem with initial conditions prescribed at another point $z_{0} \in \mathcal{D}, z_{0} \neq 0$ :

$$
\left\{\begin{array}{l}
z y^{\prime \prime}(z)+2 \Lambda y^{\prime}(z)=g(z) y(z) \quad \text { in } \mathcal{D} \\
y\left(z_{0}\right)=\tilde{y}_{0}, \quad y^{\prime}\left(z_{0}\right)=\tilde{y}_{1}
\end{array}\right.
$$

where $\tilde{y}_{0}$ and $\tilde{y}_{1}$ are complex parameters with $\tilde{y}_{0}=\mathcal{O}(1)$ and $\tilde{y}_{1}=\mathcal{O}(\Lambda)$ as $\Lambda \rightarrow \infty$. The existence and uniqueness of solution of this problem follows from Frobenius theory (when $g(z)$ is analytic at $z=z_{0}$ ) or from Picard-Lindelof's theorem (when $g(z)$ is continuous at $z=z_{0}$ ). In the following, $y_{+}(z)$ and $y_{-}(z)$ denote, respectively, the unique solutions of problems $(7)$ and (8).

When we undo the above mentioned change of unknowns, we find that $u_{ \pm}(z):=z^{\Lambda} y_{ \pm}(z)$ are a couple of independent solutions of $(5)$ whenever $\left(u_{+}\left(z_{0}\right), u_{+}^{\prime}\left(z_{0}\right)\right) \neq\left(u_{-}\left(z_{0}\right), u_{-}^{\prime}\left(z_{0}\right)\right)$. Problem (7) for $y_{+}$is equivalent to the following problem for $u_{+}$:

$$
\left\{\begin{array}{l}
z^{2} u_{+}^{\prime \prime}(z)-\Lambda(\Lambda-1) u_{+}(z)=z g(z) u_{+}(z) \quad \text { in } \mathcal{D}, \\
\lim _{z \rightarrow 0}\left[z^{-\Lambda} u_{+}(z)\right]=\bar{y}_{0}
\end{array}\right.
$$

problem that has a unique solution $u_{+}(z)$. Problem (8) for $y_{-}$is equivalent to the following problem for $u_{-}$:

$$
\left\{\begin{array}{l}
z^{2} u_{-}^{\prime \prime}(z)-\Lambda(\Lambda-1) u_{-}(z)=z g(z) u_{-}(z) \quad \text { in } \mathcal{D} \\
z_{0}^{-\Lambda} u_{-}\left(z_{0}\right)=\tilde{y}_{0}, \quad \lim _{z \rightarrow z_{0}}\left[z^{-\Lambda} u_{-}(z)\right]^{\prime}=\tilde{y}_{1}
\end{array}\right.
$$

problem that has a unique solution $u_{-}(z)$.

In the following section, for each problem, we design a sequence of functions that converges to the unique solution of the problem. For each problem, that sequence has the property of being an asymptotic sequence (not of Poincaré-type) for large $\Lambda$. In Section 5, we apply Olver's method to equation (6) and find an asymptotic expansion of Poincaré-type of two independent solutions of this equation.

\section{$3 \quad$ A fixed point method}

In this section we consider that the function $g(z)$ is continuous in the star-like domain $\mathcal{D}$. The unique solution of the initial value problem

$$
\left\{\begin{array}{l}
z \phi^{\prime \prime}(z)+2 \Lambda \phi^{\prime}(z)=0 \quad \text { in } \mathcal{D}, \\
\phi(0)=\bar{y}_{0}
\end{array}\right.
$$


is $\phi_{+}(z):=\bar{y}_{0}$. And the unique solution of the problem

$$
\left\{\begin{array}{l}
z \phi^{\prime \prime}(z)+2 \Lambda \phi^{\prime}(z)=0 \quad \text { in } \mathcal{D} \\
\phi\left(z_{0}\right)=\tilde{y}_{0}, \quad \phi^{\prime}\left(z_{0}\right)=\tilde{y}_{1}
\end{array}\right.
$$

is

$$
\phi_{-}(z):=\tilde{y}_{0}+\tilde{y}_{1} \frac{z_{0}}{1-2 \Lambda}\left[\left(\frac{z}{z_{0}}\right)^{1-2 \Lambda}-1\right] .
$$

After the change of unknown $y_{ \pm}(z) \rightarrow w_{ \pm}(z)=y_{ \pm}(z)-\phi_{ \pm}(z)$, and using (9) and (10), we find that problems (7) and (8) read, respectively,

$$
\left\{\begin{array}{l}
z w_{+}^{\prime \prime}(z)+2 \Lambda w_{+}^{\prime}(z)=F_{+}\left(z, w_{+}\right):=g(z)\left[w_{+}(z)+\phi_{+}(z)\right] \text { in } \mathcal{D} \\
w_{+}(0)=0
\end{array}\right.
$$

and

$$
\left\{\begin{array}{l}
z w_{-}^{\prime \prime}(z)+2 \Lambda w_{-}^{\prime}(z)=F_{-}\left(z, w_{-}\right):=g(z)\left[w_{-}(z)+\phi_{-}(z)\right] \text { in } \mathcal{D} \\
w_{-}\left(z_{0}\right)=w_{-}^{\prime}\left(z_{0}\right)=0 .
\end{array}\right.
$$

For convenience, we restrict the differential equations in both problems, (12) and (13) (and hence (7) and (8)), to an open straight segment $\mathcal{L} \subset \mathcal{D}$ (that may be unbounded if $\mathcal{D}$ is unbounded) with $z=0$ as an end point. Moreover, for problem (13), $z_{0} \in \mathcal{L}$ and $|z|<\left|z_{0}\right|$. See Figure 1 below.
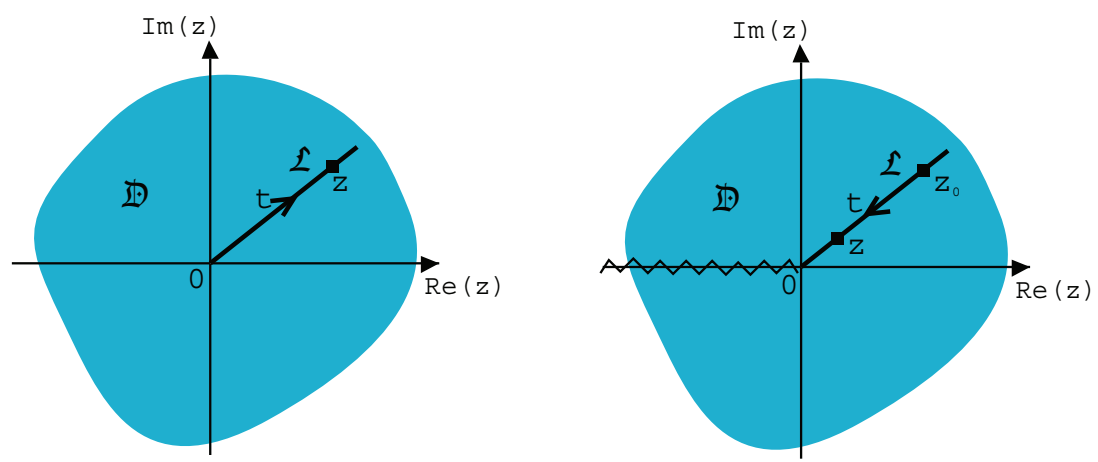

Figure 1: Domains $\mathcal{D}$ and integration paths associated to the respective problems (7) and (8). In both problems, the kernel of the operators $\mathbf{T}$ and $\tilde{\mathbf{T}}$ is bounded by 2 .

For the first problem, we seek for solutions of the equation $\mathbf{L}_{+}\left[w_{+}\right]:=z w_{+}^{\prime \prime}+2 \Lambda w_{+}^{\prime}-$ $F_{+}\left(z, w_{+}\right)$in the Banach space $\mathcal{B}_{+}:=\left\{w_{+}: \mathcal{L} \rightarrow \mathbb{C}, w_{+}(0)=0\right\}$. For the second problem, we seek for solutions of the equation $\mathbf{L}_{-}\left[w_{-}\right]:=z w_{-}^{\prime \prime}+2 \Lambda w_{-}^{\prime}-F_{-}\left(z, w_{-}\right)$in the Banach space $\mathcal{B}_{-}:=\left\{w_{-}: \mathcal{L} \rightarrow \mathbb{C}, w_{-}\left(z_{0}\right)=0\right\}$. Both spaces equipped with the sup norm:

$$
\left\|w_{ \pm}\right\|_{\infty}:=\sup _{z \in \mathcal{L}}\left|w_{ \pm}(z)\right|
$$


We write the equation $\mathbf{L}_{ \pm}\left[w_{ \pm}\right]=0$ in the form $\mathbf{L}_{ \pm}\left[w_{ \pm}\right]=\mathbf{M}\left[w_{ \pm}\right]-F_{ \pm}\left(z, w_{ \pm}\right)$, with $\mathbf{M}[w]:=$ $z w^{\prime \prime}+2 \Lambda w^{\prime}$. Then we solve the equation $\mathbf{L}_{ \pm}\left[w_{ \pm}\right]=0$ for $w_{ \pm}$using Green's function $G_{ \pm}(z, t)$ of the operator $\mathbf{M}$ with the appropriate initial conditions [7]. For problem (12), $G_{+}(z, t)$ is the unique solution of the problem

$$
\left\{\begin{array}{l}
z G_{z z}+2 \Lambda G_{z}=\delta(z-t) \quad \text { in } \mathcal{L} \\
G(0, t)=0, \quad t \in \mathcal{L}
\end{array}\right.
$$

It is given by

$$
G_{+}(z, t)=\frac{1}{2 \Lambda-1}\left[1-\left(\frac{t}{z}\right)^{2 \Lambda-1}\right] \chi_{[0, z]}(t),
$$

where $\chi_{[0, z]}(t)$ is the characteristic function of the interval $[0, z]$. For problem $(13), G_{-}(z, t)$ is the unique solution of the problem

$$
\begin{cases}z G_{z z}+2 \Lambda G_{z}=\delta(z-t) & \text { in } \mathcal{L} \\ G\left(z_{0}, t\right)=G_{z}\left(z_{0}, t\right)=0, & t, z_{0} \in \mathcal{L}\end{cases}
$$

It is given by

$$
G_{-}(z, t)=\frac{1}{2 \Lambda-1}\left[1-\left(\frac{t}{z}\right)^{2 \Lambda-1}\right] \chi_{\left[z, z_{0}\right]}(t) .
$$

Then, any solution $w_{+}(z)$ of $(12)$ is a solution of the Volterra integral equation $w_{+}(z)=$ $\left[\mathbf{T} w_{+}\right](z)$, and any solution $w_{-}(z)$ of $(13)$ is a solution of the Volterra integral equation $w_{-}(z)=$ $\left[\mathbf{T} w_{-}\right](z)$, where the integral operator $\mathbf{T}$ is defined by

$$
\left[\mathbf{T} w_{ \pm}\right](z):=\frac{1}{2 \Lambda-1} \int_{z_{0}}^{z}\left[1-\left(\frac{t}{z}\right)^{2 \Lambda-1}\right] g(t)\left[w_{ \pm}(t)+\phi_{ \pm}(t)\right] d t
$$

where $z_{0}$ must be set equal to zero for $w_{+}$. For later convenience, in the case of $w_{-}$we need to define a rescaled unknown $\tilde{w}_{-}(z):=z^{2 \Lambda-1} w_{-}(z)$ and consider the rescaled operator

$$
\left[\tilde{\mathbf{T}} \tilde{w}_{-}\right](z):=\frac{1}{2 \Lambda-1} \int_{z_{0}}^{z}\left[\left(\frac{z}{t}\right)^{2 \Lambda-1}-1\right] g(t)\left[\tilde{w}_{-}(t)+\tilde{\phi}_{-}(t)\right] d t
$$

with $\tilde{\phi}_{-}(z):=z^{2 \Lambda-1} \phi_{-}(z)$.

For any complex $z$ in $\mathcal{L}$, the kernel $1-(t / z)^{2 \Lambda-1}$ of $\mathbf{T}$, is uniformly bounded in $t \in[0, z]$ by 2 , independently of $\Lambda$ and $z$. Also, for any complex $z$ in $\mathcal{L}$, with $|z|<\left|z_{0}\right|$, the kernel $(z / t)^{2 \Lambda-1}-1$ of $\tilde{\mathbf{T}}$, is uniformly bounded in $t \in\left[z, z_{0}\right]$ by 2 , independently of $\Lambda$ and $z$.

From the Banach fixed point theorem [1, pp. 26, Theorem 3.1] it is well known that, if any power of the operator $\mathbf{T}$ is contractive in $\mathcal{B}_{+}$, then the equation $w_{+}(z)=\left[\mathbf{T} w_{+}\right](z)$ has a unique solution $w_{+}(z)$ (fixed point of $\mathbf{T}$ ) and the sequence $w_{n+1}^{+}=\left[\mathbf{T} w_{n}^{+}\right], w_{0}^{+}=0$, converges to that solution $w_{+}(z)$. Analogously, if any power of the operator $\tilde{\mathbf{T}}$ is contractive in $\mathcal{B}_{-}$, then the equation $\tilde{w}_{-}(z)=\left[\tilde{\mathbf{T}} \tilde{w}_{-}\right](z)$ has a unique solution $\tilde{w}_{-}(z)$ (fixed point of $\tilde{\mathbf{T}}$ ) and the sequence $\tilde{w}_{n+1}^{-}=\left[\tilde{\mathbf{T}} \tilde{w}_{n}^{-}\right], \tilde{w}_{0}^{-}=0$, converges to that solution $\tilde{w}_{-}(z)$. 
We show this for the operator $\tilde{\mathbf{T}}$. The proof for the operator $\mathbf{T}$ is identical replacing $z_{0}$ by 0 . It is straightforward to show the contractive character of the operator $\tilde{\mathbf{T}}$ : from its definition we have that, for any couple $u, v \in \mathcal{B}_{-}$,

$$
|[\tilde{\mathbf{T}} u](z)-[\tilde{\mathbf{T}} v](z)| \leq \frac{2}{|2 \Lambda-1|} \int_{z_{0}}^{z}|g(t)||u(t)-v(t)||d t| \leq\left|\frac{2\left(z-z_{0}\right)}{2 \Lambda-1}\right|\|g\|_{\infty}\|u-v\|_{\infty} .
$$

We also have

$$
\begin{aligned}
\left|\left[\tilde{\mathbf{T}}^{2} u\right](z)-\left[\tilde{\mathbf{T}}^{2} v\right](z)\right| & \leq \frac{2}{|2 \Lambda-1|} \int_{z_{0}}^{z}|g(t)\|[\tilde{\mathbf{T}} u](t)-[\tilde{\mathbf{T}} v](t)\| d t| \\
& \leq\left|\frac{\left[2\left(z-z_{0}\right)\right]^{2}}{2(2 \Lambda-1)^{2}}\right|\|g\|_{\infty}^{2}\|u-v\|_{\infty}
\end{aligned}
$$

and

$$
\begin{aligned}
\left|\left[\tilde{\mathbf{T}}^{3} u\right](z)-\left[\tilde{\mathbf{T}}^{3} v\right](z)\right| & \leq \frac{2}{|2 \Lambda-1|} \int_{z_{0}}^{z}|g(t)|\left|\left[\tilde{\mathbf{T}}^{2} u\right](t)-\left[\tilde{\mathbf{T}}^{2} v\right](t) \| d t\right| \\
& \leq\left|\frac{\left.2\left(z-z_{0}\right)\right]^{3}}{3 !(2 \Lambda-1)^{3}}\right|\|g\|_{\infty}^{3}\|u-v\|_{\infty} .
\end{aligned}
$$

It is straightforward to prove, by means of induction over $n$ that, for $n=1,2,3, \ldots$,

$$
\left|\left[\tilde{\mathbf{T}}^{n} u\right](z)-\left[\tilde{\mathbf{T}}^{n} v\right](z)\right| \leq\left|\frac{\left(2\left(z-z_{0}\right)\right)^{n}}{n !(2 \Lambda-1)^{n}}\right|\|g\|_{\infty}^{n}\|u-v\|_{\infty} .
$$

This means that, for bounded $z$, the operators $\mathbf{T}^{n}$ and $\tilde{\mathbf{T}}^{n}$ are contractive for large enough $n$. From $\left[1\right.$, pp. 26, Theorem 3.1] we have that the sequence $w_{n+1}^{+}=\left[\mathbf{T} w_{n}^{+}\right], n=0,1,2, \ldots$, $w_{0}^{+}=0$, converges, for any $z \in \mathcal{L}$ bounded, to the unique solution $w_{+}(z)$ of problem (12) and the sequence $\tilde{w}_{n+1}^{-}=\left[\tilde{\mathbf{T}} \tilde{w}_{n}^{-}\right], n=0,1,2, \ldots, \tilde{w}_{0}^{-}=0$, converges, for any $z \in \mathcal{L}$ bounded, to the unique solution $w_{-}(z)$ of problem (13) multiplied by $z^{2 \Lambda-1}$. Or equivalently, the sequence $y_{n}^{+}:=w_{n}^{+}+\phi_{+}$, that is,

$$
y_{n+1}^{+}(z)=\bar{y}_{0}+\frac{z}{2 \Lambda-1} \int_{0}^{1}\left[1-t^{2 \Lambda-1}\right] g(z t) y_{n}^{+}(z t) d t, \quad y_{0}^{+}(z)=\bar{y}_{0},
$$

converges, for $z \in \mathcal{L}$ bounded, to the unique solution $y_{+}(z)$ of $(7)$. And the sequence $y_{n}^{-}:=$ $w_{n}^{-}+\phi_{-}$, with $w_{n}^{-}:=z^{1-2 \Lambda} \tilde{w}_{n}^{-}$, that is,

$$
y_{n+1}^{-}(z)=\phi_{-}(z)+\frac{1}{2 \Lambda-1} \int_{z_{0}}^{z}\left[1-\left(\frac{t}{z}\right)^{2 \Lambda-1}\right] g(t) y_{n}^{-}(t) d t, \quad y_{0}^{-}(z)=\phi_{-}(z),
$$

converges, for $z \in \mathcal{L}$ bounded, to the unique solution $y_{-}(z)$ of (8).

Let's define the remainder of the approximation by $R_{n}^{ \pm}(z):=y_{ \pm}(z)-y_{n}^{ \pm}(z)$. Setting $v(z)=$ $w_{+}(z)$ and $u(z)=w_{0}^{+}(z)=0$ in (14) and using that $\left[\mathbf{T}^{n} w_{+}\right]=w_{+}$and $\left[\mathbf{T}^{n} w_{0}^{+}\right]=w_{n}^{+}$or setting $v(z)=w_{-}(z)$ and $u(z)=w_{0}^{-}(z)=0$ in (14) and using that $\left[\tilde{\mathbf{T}}^{n} \tilde{w}_{-}\right]=\tilde{w}_{-}$and $\left[\tilde{\mathbf{T}}^{n} \tilde{w}_{0}^{-}\right]=\tilde{w}_{n}^{-}$we find

$$
\left|w_{ \pm}(z)-w_{n}^{ \pm}(z)\right| \leq \frac{\|g\|_{\infty}^{n}\left|\left[2\left(z-z_{0}\right)\right]^{n}\right|}{n !|2 \Lambda-1|^{n}}|| w_{ \pm} \|_{\infty} .
$$


In this formula and formulas below involving $w^{+}$or $y^{+}$(not $w^{-}$or $y^{-}$) we must set $z_{0}=0$. Using that $y_{ \pm}(z)=w_{ \pm}(z)+\phi_{ \pm}(z)$ and $y_{n}^{ \pm}(z)=w_{n}^{ \pm}(z)+\phi_{ \pm}(z)$ we find that the remainder $R_{n}^{ \pm}(z)$ is bounded by

$$
\left|R_{n}^{ \pm}(z)\right| \leq \frac{\|g\|_{\infty}^{n}\left|\left[2\left(z-z_{0}\right)\right]^{n}\right|}{n !|2 \Lambda-1|^{n}}\left\|y_{ \pm}-\phi_{ \pm}\right\|_{\infty} .
$$

Moreover, we have that, for problem (7),

$$
y_{n+1}^{+}(z)-y_{n}^{+}(z)=\frac{z}{2 \Lambda-1} \int_{0}^{1}\left[1-t^{2 \Lambda-1}\right] g(z t)\left[y_{n}^{+}(z t)-y_{n-1}^{+}(z t)\right] d t,
$$

and, for problem (8),

$$
y_{n+1}^{-}(z)-y_{n}^{-}(z)=\frac{1}{2 \Lambda-1} \int_{z_{0}}^{z}\left[1-\left(\frac{t}{z}\right)^{2 \Lambda-1}\right] g(t)\left[y_{n}^{-}(t)-y_{n-1}^{-}(t)\right] d t .
$$

Then, for any problem,

$$
\left\|y_{n+1}^{ \pm}-y_{n}^{ \pm}\right\|_{\infty} \leq \frac{2\left|z-z_{0}\right|\|g\|_{\infty}}{|2 \Lambda-1|}\left\|y_{n}^{ \pm}-y_{n-1}^{ \pm}\right\|_{\infty} .
$$

This means that the expansion

$$
y^{ \pm}(z)=\phi_{ \pm}+\sum_{k=0}^{n-1}\left[y_{k+1}^{ \pm}(z)-y_{k}^{ \pm}(z)\right]+R_{n}^{ \pm}(z)
$$

is an asymptotic expansion for large $\Lambda$ and bounded $z \in \mathcal{L}$.

We see from $(15)$ that the sequence $y_{n}^{+}(z)$ is a sequence of analytic functions in $\mathcal{D}$. A sequence of analytic functions that converges uniformly in any compact contained in $\mathcal{D}$, that is, the unique solution $y_{+}(z)$ of problem $(7)$ is analytic in $\mathcal{D}$. Analogously, the sequence $y_{n}^{-}(z)$ in (16) is a sequence of analytic functions in $\mathcal{D}$ with, possibly, a branch point at $z=0$. This means that the unique solution $y_{-}(z)$ of problem (8) is analytic in $\mathcal{D}$ except, possibly, for a branch point at $z=0$.

Observation 1. When $g(z)$ is not analytic in $\mathcal{D}$, but only continuous, from the above derivation we still see that, problems (7) and (8) have a unique solution and the recurrences (15) and (16) converge to the respective solutions.

Observation 2. When $g(z)$ is an elementary function (analytic or not in $\mathcal{D}$ ), the successive approximations $y_{n}$ of the unique solution of those problems are iterated integrals of elementary functions.

\section{The nonlinear case}

The technique used in the previous section may be easily generalized to nonlinear problems of the form

$$
u^{\prime \prime}-\frac{\tilde{\Lambda}^{2}}{z^{2}} u=\tilde{f}(z, u), \quad \tilde{\Lambda} \rightarrow \infty
$$


where the function $\tilde{f}(z, u)$ is continuous for $(z, y) \in \mathcal{D} \times \mathbb{C}$ and satisfies the following Lipschitz condition in its second variable:

$$
|\tilde{f}(z, u)-\tilde{f}(z, v)| \leq \frac{L}{z}|u-v|, \quad \forall u, v \in \mathbb{C} \text { and } z \in \mathcal{D},
$$

with $L$ a positive constant independent of $z, u, v$ and $\mathcal{D}$ is a star-like domain.

After the change of unknown: $u \rightarrow y:=z^{-\Lambda} u$, with the parameter $\Lambda$ defined in (4), the new unknown $y$ satisfies the nonlinear differential equation

$$
z y^{\prime \prime}(z)+2 \Lambda y^{\prime}(z)=f(z, y(z), \Lambda)
$$

where $f(z, y, \Lambda):=z^{1-\Lambda} \tilde{f}\left(z, z^{\Lambda} y\right)$. Then, two possible well-posed problems, each of one provides a unique solution of the equation (19), are

$$
\left\{\begin{array}{l}
z y^{\prime \prime}(z)+2 \Lambda y^{\prime}(z)=f(z, y(z), \Lambda) \text { in } \mathcal{D}, \\
y(0)=\bar{y}_{0}
\end{array}\right.
$$

and

$$
\left\{\begin{array}{l}
z y^{\prime \prime}(z)+2 \Lambda y^{\prime}(z)=f(z, y(z), \Lambda) \quad \text { in } \mathcal{D}, \\
y\left(z_{0}\right)=\tilde{y}_{0}, \quad y^{\prime}\left(z_{0}\right)=\tilde{y}_{1},
\end{array}\right.
$$

where $z_{0} \neq 0, \bar{y}_{0}=\mathcal{O}(1), \tilde{y}_{0}=\mathcal{O}(1)$ and $\tilde{y}_{1}=\mathcal{O}(\Lambda)$ are complex numbers.

A slight modification of the analysis of Section 3 provides, for problems (20) and (21) the same conclusions that we derived for problems (7) and (8). We state them in the form of a theorem.

Theorem 1. Let $f: \mathcal{D} \times \mathbb{C} \rightarrow \mathbb{C}$ continuous and satisfy (18). Then, problems (20) and (21) have unique solutions that we denote by $y_{+}(z)$ and $y_{-}(z)$ respectively. They are independent whenever $\left(y_{+}\left(z_{0}\right), y_{+}^{\prime}\left(z_{0}\right)\right) \neq\left(y_{-}\left(z_{0}\right), y_{-}^{\prime}\left(z_{0}\right)\right)$. Moreover:

(i) For $n=0,1,2, \ldots$, the sequences

$$
\begin{gathered}
y_{n+1}^{+}(z)=\bar{y}_{0}+\frac{z}{2 \Lambda-1} \int_{0}^{1}\left[1-t^{2 \Lambda-1}\right] f\left(t z, y_{n}^{+}(z t), \Lambda\right) d t, \quad y_{0}^{+}(z)=\bar{y}_{0}, \\
y_{n+1}^{-}(z)=\phi_{-}(z)+\frac{1}{2 \Lambda-1} \int_{z_{0}}^{z}\left[1-\left(\frac{t}{z}\right)^{2 \Lambda-1}\right] f\left(t, y_{n}^{-}(t), \Lambda\right) d t, \quad y_{0}^{-}(z)=\phi_{-}(z),
\end{gathered}
$$

with $\phi_{-}(z)$ defined in (11), converge, for $z \in \mathcal{L}$ bounded, to the unique solutions $y_{+}(z)$ of (20) and $y_{-}(z)$ of (21) respectively.

(ii) The remainder $R_{n}^{ \pm}(z):=y_{ \pm}(z)-y_{n}^{ \pm}(z)$ is bounded by

$$
\left|R_{n}^{ \pm}(z)\right| \leq \frac{L^{n}\left|\left[2\left(z-z_{0}\right)\right]^{n}\right|}{n !|2 \Lambda-1|^{n}}|| y_{ \pm}-\phi_{ \pm} \|_{\infty} .
$$

And, in consequence, the expansion

$$
y^{ \pm}(z)=\phi_{ \pm}+\sum_{k=0}^{n-1}\left[y_{k+1}^{ \pm}(z)-y_{k}^{ \pm}(z)\right]+R_{n}^{ \pm}(z)
$$

is an asymptotic expansion for large $\Lambda$ and bounded $z \in \mathcal{L}$. 
Proof. It is similar to the analysis of the previous section. Then, we only give here a few significant details. After the change of unknown $y_{ \pm}(z) \rightarrow w_{ \pm}(z):=y_{ \pm}(z)-\phi_{ \pm}(z)$, problems (20), (21) read, respectively

$$
\left\{\begin{array}{l}
z w_{+}^{\prime \prime}(z)+2 \Lambda w_{+}^{\prime}(z)=F_{+}\left(z, w_{+}\right):=f\left(z, w_{+}(z)+\phi_{+}(z), \Lambda\right) \text { in } \mathcal{D} \\
w_{+}(0)=0
\end{array}\right.
$$

and

$$
\left\{\begin{array}{l}
z w_{-}^{\prime \prime}(z)+2 \Lambda w_{-}^{\prime}(z)=F_{-}\left(z, w_{-}\right):=f\left(z, w_{-}(z)+\phi_{-}(z), \Lambda\right) \text { in } \mathcal{D} \\
w_{-}\left(z_{0}\right)=w_{-}^{\prime}\left(z_{0}\right)=0 .
\end{array}\right.
$$

The solutions of these problems satisfy the Volterra integral equations of the second kind $w_{+}(z)=$ $\left[\mathbf{T} w_{+}\right](z)$ and $w_{-}(z)=\left[\mathbf{T} w_{-}\right](z)$ where now, the operator $\mathbf{T}$ is nonlinear, and defined by

$$
\left[\mathbf{T} w_{ \pm}\right](z):=\frac{1}{2 \Lambda-1} \int_{z_{0}}^{z}\left[1-\left(\frac{t}{z}\right)^{2 \Lambda-1}\right] f\left(t, w_{ \pm}(t)+\phi_{ \pm}(t), \Lambda\right) d t
$$

where $z_{0}$ must be set equal to zero for $w_{+}$. From (18) we have the Lipschitz condition

$$
|f(z, u, \Lambda)-f(z, v, \Lambda)| \leq L|u-v|, \quad \forall u, v \in \mathbb{C} \text { and } z \in \mathcal{D},
$$

with $L$ given in (18). From here, and using (22) the proof is identical to the one of the previous section replacing $\|g\|_{\infty}$ by $L$.

\section{Olver's method for equation (6)}

In this section we assume that the function $g(z)$ is infinitely differentiable in the star-like domain $\mathcal{D}$. Consider two (at this moment unknown) independent solutions $Y_{+}(z)$ and $Y_{-}(z)$ of $(6)$ and propose the following representations in the form of formal asymptotic expansions for large $\Lambda$ :

$$
Y_{+}(z)=Y_{n}^{+}(z)+R_{n}^{+}(z), \quad Y_{-}(z)=Y_{n}^{-}(z)+z^{1-2 \Lambda} R_{n}^{-}(z)
$$

with

$$
Y_{n}^{+}(z):=\sum_{k=0}^{n-1} \frac{A_{k}(z)}{(2 \Lambda)^{k}}, \quad Y_{n}^{-}(z):=z^{1-2 \Lambda} \sum_{k=0}^{n-1} \frac{A_{k}(z)}{[2(1-\Lambda)]^{k}},
$$

and the obvious definition of $R_{n}^{ \pm}(z)$. When we introduce (23) and (24) in the equation $z y^{\prime \prime}+$ $2 \Lambda y^{\prime}=g y$ we find that both, $Y_{+}(z)$ and $Y_{-}(z)$, formally satisfy the respective differential equations, term-wise in $(2 \Lambda)^{k}$ or $[2(1-\Lambda)]^{k}$, if, for $n=0,1,2, \ldots$,

$$
A_{n+1}(z)=A_{n}(z)-z A_{n}^{\prime}(z)+\int_{0}^{z} g(t) A_{n}(t) d t
$$

and

$$
\begin{gathered}
z\left[R_{n}^{+}(z)\right]^{\prime \prime}+2 \Lambda\left[R_{n}^{+}(z)\right]^{\prime}=\frac{A_{n}^{\prime}(z)}{(2 \Lambda)^{n-1}}+g(z) R_{n}^{+}(z), \\
z\left[R_{n}^{-}(z)\right]^{\prime \prime}+2(1-\Lambda)\left[R_{n}^{-}(z)\right]^{\prime}=\frac{A_{n}^{\prime}(z)}{[2(1-\Lambda)]^{n-1}}+g(z) R_{n}^{-}(z) .
\end{gathered}
$$


Without loss of generality we may fix $A_{0}(z)=1$.

We seek a solution $Y^{+}(z)$ regular at $z=0$ and a solution $Y^{-}(z)$ regular at $z=z_{0} \neq 0$. Therefore, without loss of generality, we may set $R_{n}^{+}(0)=0$ and $R_{n}^{-}\left(z_{0}\right)=\left[R_{n}^{-}\right]^{\prime}\left(z_{0}\right)=0$. Then, these remainders are solutions of the respective initial value problems:

$$
\left\{\begin{array}{l}
z\left[R_{n}^{+}(z)\right]^{\prime \prime}+2 \Lambda\left[R_{n}^{+}(z)\right]^{\prime}=\frac{A_{n}^{\prime}(z)}{(2 \Lambda)^{n-1}}+g(z) R_{n}^{+}(z) \text { in } \mathcal{D} \\
R_{n}^{+}(0)=0
\end{array}\right.
$$

and

$$
\left\{\begin{array}{l}
z\left[R_{n}^{-}(z)\right]^{\prime \prime}+2(1-\Lambda)\left[R_{n}^{-}(z)\right]^{\prime}=\frac{A_{n}^{\prime}(z)}{[2(1-\Lambda)]^{n-1}}+g(z) R_{n}^{-}(z) \text { in } \mathcal{D} \\
R_{n}^{-}\left(z_{0}\right)=\left[R_{n}^{-}\right]^{\prime}\left(z_{0}\right)=0 .
\end{array}\right.
$$

The first problem for $R_{n}^{+}(z)$ is identical to problem (12) for $w^{+}(z)$ replacing $g(z) \phi_{+}(z)$ by $A_{n}^{\prime}(z) /(2 \Lambda)^{n-1}$. The second problem for $R_{n}^{-}(z)$ is identical to problem (13) for $w^{-}(z)$ replacing $g(z) \phi_{-}(z)$ by $A_{n}^{\prime}(z) /(2 \Lambda)^{n-1}$ and then $\Lambda$ by $1-\Lambda$. Therefore, proceeding as in Section 3 we find that $R_{n}^{+}(z)$ and $R_{n}^{-}(z)$ are solutions of the respective Volterra integral equations

$$
\begin{gathered}
R_{n}^{+}(z)=\frac{1}{2 \Lambda-1} \int_{0}^{z}\left[1-\left(\frac{t}{z}\right)^{2 \Lambda-1}\right]\left[\frac{A_{n}^{\prime}(t)}{(2 \Lambda)^{n-1}}+g(t) R_{n}^{+}(t)\right] d t, \\
R_{n}^{-}(z)=\frac{1}{1-2 \Lambda} \int_{z_{0}}^{z}\left[1-\left(\frac{z}{t}\right)^{2 \Lambda-1}\right]\left[\frac{A_{n}^{\prime}(t)}{[2(1-\Lambda)]^{n-1}}+g(t) R_{n}^{-}(t)\right] d t .
\end{gathered}
$$

Using that $\left|1-(t / z)^{2 \Lambda-1}\right| \leq 2$ for $t \in[0, z]$ and $\left|1-(z / t)^{2 \Lambda-1}\right| \leq 2$ for $t \in\left[z, z_{0}\right]$, we derive the bound

$$
\left|R_{n}^{-}(z)\right| \leq \frac{2}{|2 \Lambda-1|} \int_{z_{0}}^{z}\left|g(t) R_{n}^{-}(t)\right||d t|+\frac{2}{|2 \Lambda-1|} \int_{z_{0}}^{z}\left|\frac{A_{n}^{\prime}(t)}{[2(1-\Lambda)]^{n-1}}\right||d t|
$$

and the same bound for $R_{n}^{+}(z)$ replacing $\Lambda$ by $1-\Lambda$ and setting $z_{0}=0$. Applying Gronwall's lemma [2] we obtain

$$
\left|R_{n}^{-}(z)\right| \leq \frac{2 e^{\frac{2}{|2 \Lambda-1|} \int_{z_{0}}^{z}|g(t)||d t|}}{\left|(2 \Lambda-1)[2(1-\Lambda)]^{n-1}\right|} \int_{z_{0}}^{z}\left|A_{n}^{\prime}(t)\right||d t|
$$

and the same bound for $R_{n}^{+}(z)$ replacing $\Lambda$ by $1-\Lambda$ and setting $z_{0}=0$. When $A_{n}^{\prime}(t)$ and $g(t)$ are integrable in $\mathcal{L}$ (this is granted when $\mathcal{L}$ is bounded), we also have the bounds:

$$
\begin{array}{r}
\left|R_{n}^{+}(z)\right| \leq \frac{2\left\|A_{n}^{\prime}\right\|_{1}}{\left|(2 \Lambda-1)(2 \Lambda)^{n-1}\right|} e^{2\|g\|_{1} /|2 \Lambda-1|}, \\
\left|R_{n}^{-}(z)\right| \leq \frac{2\left\|A_{n}^{\prime}\right\|_{1}}{\left|(2 \Lambda-1)[2(1-\Lambda)]^{n-1}\right|} e^{2\|g\|_{1} /|2 \Lambda-1|},
\end{array}
$$

where

$$
\|g\|_{1}:=\int_{\mathcal{L}}\left|g(t)\left\|d t\left|, \quad\left\|A_{n}^{\prime}\right\|_{1}:=\int_{\mathcal{L}}\right| A_{n}^{\prime}(t)\right\| d t\right| .
$$

These bounds show the asymptotic character of the expansions (23). 
Observation 3. The unique solution $y_{-}(z)$ of problem (8) is approximated by $y_{n}^{-}(z):=$ $a_{n} Y_{n}^{+}(z)+b_{n} Y_{n}^{-}(z)$, where the coefficients $a_{n}$ and $b_{n}$ must be approximated at any order $n$ of the approximation by using the conditions $y_{-}\left(z_{0}\right)=\tilde{y}_{0}$ and $y_{-}^{\prime}\left(z_{0}\right)=\tilde{y}_{1}$, and depend on the function $g, \Lambda$ and the point $z_{0}$. The situation is simpler for the unique solution $y_{+}(z)$ of problem (7). It is approximated by $y_{n}^{+}(z):=c_{n} Y_{n}^{+}(z)$, where the coefficient $c_{n}$ must be approximated at any order $n$ of the approximation by using the condition $y_{+}(0)=\bar{y}_{0}$. It is easy to see that $A_{n}(0)=1$ for $n=0,1,2, \ldots$ Then, when we impose the condition $y_{n}^{+}(0)=\bar{y}_{0}$, we find that the coefficient $c_{n}$ is indeed independent of the function $g(z)$ :

$$
c_{n}=\bar{y}_{0} \frac{(2 \Lambda)^{n-1}(2 \Lambda-1)}{(2 \Lambda)^{n}-1},
$$

and is of order $\mathcal{O}(1)$ as $|\Lambda| \rightarrow \infty$.

Observation 4. We see from (25) that the coefficients $A_{n}(z), n=0,1,2, \ldots$, are infinitely differentiable in $\mathcal{D}$. Moreover, when $g(z)$ is analytic in $\mathcal{D}$, the coefficients $A_{n}(z), n=0,1,2, \ldots$, are analytic in $\mathcal{D}$ too.

\section{Example and numerical experiments}

Consider the differential equation

$$
z y^{\prime \prime}(z)+2 \Lambda y^{\prime}(z)=y(z) .
$$

To find asymptotic approximations for large $\Lambda$ of two independent solutions of this equation we consider the two associated initial value problems:

$$
\left\{\begin{array}{l}
z y^{\prime \prime}(z)+2 \Lambda y^{\prime}(z)=y(z) \text { in } \mathbb{C}, \\
y(0)=1
\end{array}\right.
$$

and

$$
\left\{\begin{array}{l}
z y^{\prime \prime}(z)+2 \Lambda y^{\prime}(z)=y(z) \quad \text { in } \mathbb{C}, \\
y(1)=K_{2 \Lambda-1}(2), \quad y^{\prime}(1)=-K_{2 \Lambda}(2) .
\end{array}\right.
$$

The unique solution of $(28)$ is a modified Bessel function (analytic in $\mathbb{C}$ )

$$
y_{+}(z)=\Gamma(2 \Lambda) z^{1 / 2-\Lambda} I_{2 \Lambda-1}(2 \sqrt{z}),
$$

and the unique solution of (29) is a modified Bessel function

$$
y_{-}(z)=z^{1 / 2-\Lambda} K_{2 \Lambda-1}(2 \sqrt{z}),
$$

analytic in $\mathbb{C} \backslash \mathbb{R}^{-}$.

The iterative method introduced in Section 3 provides a convergent as well as an asymptotic expansion of these functions for large $\Lambda$ in terms of elementary functions. The recurrence relation (15) for problem (28) is given by

$$
\begin{aligned}
& y_{0}^{+}(z)=1, \\
& y_{n+1}^{+}(z)=1+\frac{z}{2 \Lambda-1} \int_{0}^{1}\left[1-t^{2 \Lambda-1}\right] y_{n}^{+}(z t) d t,
\end{aligned}
$$


and the recurrence relation (16) for problem (29) is defined by

$$
\begin{aligned}
& y_{0}^{-}(z)=K_{1-2 \Lambda}(2)-\frac{K_{2 \Lambda}(2)}{1-2 \Lambda}\left(z^{1-2 \Lambda}-1\right) \\
& y_{n+1}^{-}(z)=y_{0}^{-}(z)+\frac{1}{2 \Lambda-1} \int_{z_{0}}^{z}\left[1-\left(\frac{t}{z}\right)^{2 \Lambda-1}\right] y_{n}^{-}(t) d t .
\end{aligned}
$$

It is noteworthy that $y_{n}^{+}(z), n=0,1,2, \ldots$, are just the partial sums of the power series expansion of $y_{+}(z)$ [6, Sec. 25, p. 249, eq. 10.25.2]:

$$
y_{n}^{+}(z)=\sum_{k=0}^{n} \frac{z^{k}}{k !(2 \Lambda)_{k}} .
$$

On the other hand, applying Olver's method as it is specified in Observation 3, we know that an asymptotic approximation of the order $n$ of the unique solution $y_{+}(z)$ of problem (28) is $y_{n}^{+}(z)=c_{n} Y_{n}^{+}(z)$, with $c_{n}$ given in (27) and $Y_{n}^{+}(z)$ in (24). An asymptotic approximation of the order $n$ of the unique solution $y_{-}(z)$ of problem (29) is $y_{n}^{-}(z)=a_{n} Y_{n}^{+}(z)+b_{n} Y_{n}^{-}(z)$, with $Y_{n}^{+}(z)$ and $Y_{n}^{-}(z)$ given in (24). The coefficients $a_{n}$ and $b_{n}$ are computed at any order $n$ of the approximation by solving the algebraic system of two equations that we obtain when we impose the conditions $y(1)=K_{2 \Lambda-1}(2)$ and $y^{\prime}(1)=-K_{2 \Lambda}(2)$.

From (25) with $g(z)=1$ we find:

$$
\left\{\begin{array}{l}
A_{0}(z)=1 \\
A_{n+1}(z)=A_{n}(z)-z A_{n}^{\prime}(z)+\int_{0}^{z} A_{n}(t) d t .
\end{array}\right.
$$

They are polynomials in the variable $z$ :

$$
\begin{aligned}
& A_{0}(z)=1, \quad A_{1}(z)=1+z, \quad A_{2}(z)=1+z+\frac{z^{2}}{2}, \quad A_{3}(z)=1+z+\frac{z^{3}}{6}, \\
& A_{4}(z)=1+z+\frac{z^{2}}{2}-\frac{z^{3}}{3}+\frac{z^{4}}{24}, \quad A_{5}(z)=1+z+\frac{5 z^{3}}{6}-\frac{5 z^{4}}{24}+\frac{z^{5}}{120}, \quad \ldots
\end{aligned}
$$

Thus, Olver's method also gives an asymptotic expansion of the unique solution $y_{+}(z)$ of (28) and the unique solution $y_{-}(z)$ of $(29)$ for large $|\Lambda|$ in terms of elementary functions of $z$.

Table 1 and Table 2 show some numerical approximations, for different values of $z$ and $\Lambda$, of the solutions of (28) and (29) respectively supplied by the iterative algorithm compared with the approximation given by Olver's method.

\section{$7 \quad$ Final remarks}

As Olver remarks in [5, Chap. 12, p. 475, Theorem 14.1], Olver's asymptotic expansion (2) does not work for $m=2$. In Section 2 we have modified the differential equation in the case $m=2$ that moves the asymptotic parameter $\Lambda$ from the coefficient of the unknown $u$ in the original differential equation to the coefficient of the derivative $y^{\prime}$ in the new differential equation. Then, we have proposed two methods to obtain asymptotic expansions of two independent solutions 


\begin{tabular}{c|c|c|c|}
\hline \multicolumn{3}{|c}{$z=1$} \\
\hline \hline$\Lambda$ & $n$ & Olver's method & Formula (30) \\
\hline \multirow{3}{*}{0.75} & 1 & 0.22798242 & 0.080931451 \\
& 3 & 0.06396403 & 0.00040353 \\
& 5 & 0.01879412 & $3.69 e-7$ \\
\hline \multirow{5}{*}{5} & 1 & 0.01246076 & 0.00423127 \\
& 3 & 0.00010294 & $2.22 e-6$ \\
& 5 & $5.66 e-7$ & $3.52 e-10$ \\
\hline \multirow{5}{*}{100} & 1 & 0.00003714 & 0.00001239 \\
& 3 & $7.49 e-10$ & $2.51 e-11$ \\
& 5 & $9.25 e-15$ & $2.00 e-17$ \\
\hline \multirow{3}{*}{500} & 1 & $1.49 e-6$ & $4.99 e-7$ \\
& 3 & $1.20 e-12$ & $4.13 e-14$ \\
& 5 & $5.93 e-19$ & $1.0 e-21$ \\
\hline
\end{tabular}

\begin{tabular}{c|c|c|c|}
\hline \multicolumn{4}{|c}{$z=-2$} \\
\hline \hline$\Lambda$ & $n$ & Olver's method & Formula (30) \\
\hline \multirow{3}{*}{0.5} & 1 & 1.00000000 & 4.08781323 \\
& 3 & 0.69593774 & 0.13062516 \\
& 5 & 6.00987600 & 0.00060326 \\
\hline \multirow{3}{*}{5} & 1 & 0.00118982 & 0.02105883 \\
& 3 & 0.00027873 & 0.00004621 \\
& 5 & 0.00002455 & $2.96 e-8$ \\
\hline \multirow{3}{*}{$50-2 i$} & 1 & $1.31 e-6$ & 0.00020038 \\
& 3 & $3.25 e-8$ & $6.36 e-9$ \\
& 5 & $2.8 e-11$ & $1.1 e-13$ \\
\hline \multirow{3}{*}{100} & 1 & $1.65 e-7$ & 0.00005008 \\
& 3 & $2.06 e-9$ & $4.07 e-10$ \\
& 5 & $5.0 e-13$ & $1.0 e-14$ \\
\hline
\end{tabular}

Table 1: Numerical experiments about the relative errors in the approximation of the solution of problem (28) using Olver's method and the iterative method (30) for different values of $\Lambda$ and $n$. For the given values of $n$, the relative errors correspond to the approximate solution $y_{n}^{+}(z)$ for the iterative method and the approximate solution $c_{n+1} Y_{n+1}^{+}(z)$ for Olver's method.

of this equation: one method is just Olver's idea applied to the new differential equation. The other method is a fixed point technique that gives an asymptotic expansion for large $\Lambda$ that is also convergent. Moreover, this second method can be also applied to nonlinear differential equations. For $m \neq 2$ the asymptotic behavior for large $\Lambda$ of the solutions of (1) is exponential. As a difference with the cases $m \neq 2$, in the case $m=2$ the asymptotic behavior of the solutions is not exponential, but of power type. This is why the standard Olver's method cannot be directly applied in this case.

The approximations $y_{n}^{+}(z)$ to the unique solution $y_{+}(z)$ of problem $(7)$, derived with either, the fixed point method of Section 3, or Olver's method of Section 5, are analytic in $\mathcal{D}$ when $g(z)$ is analytic. On the other hand, the approximation $y_{n}^{-}(z)$ to the unique solution $y_{-}(z)$ of problem (8), derived with either, the fixed point method or Olver's method are analytic in $\mathcal{D}$ when $g(z)$ is analytic there, except, possibly, for a branch point at $z=0$. In fact, when $g(z)$ is analytic in $\mathcal{D}$, the solution $y_{+}(z)$ of $(7)$ is analytic in $\mathcal{D}$, whereas the solution $y_{-}(z)$ of $(8)$ is analytic in $\mathcal{D}$ except, possibly, for a branch point at $z=0$. The difference between the approximations given by Olver's method and the approximations given by the fixed point method is that the later are convergent, whereas the former, in general, are not. Then, the analytic properties of the solution are the same as the analytic properties of the approximants in both methods. Also, in Olver's method, the remainder $R_{n}^{+}(z)$ is analytic in $\mathcal{D}$, whereas the remainder $R_{n}^{-}(z)$ is analytic in $\mathcal{D}$ except, possibly, for a branch point at $z=0$. Another difference between the approximations supplied by the iterative and Olver's technique is the following. The iterative technique gives the approximations $y_{n}^{+}(z)$ and $y_{n}^{-}(z)$ to the unique solutions of the respective problems $(7)$ and (8) directly, from algorithm (15) and (16). On the other hand, Olver's technique gives, in a 


\begin{tabular}{c|c|c|c|}
\hline \multicolumn{4}{|c}{$z=0.5$} \\
\hline \hline \multirow{3}{*}{0.75} & $n$ & Olver's method & Formula (31) \\
& 1 & 0.11724359 & 0.00308515 \\
& 3 & 0.15072603 & $2.04 e-7$ \\
& 5 & 0.22999718 & $1.98 e-12$ \\
\hline \multirow{5}{*}{5} & 1 & 0.04701568 & 0.00080406 \\
& 3 & 0.00120818 & $3.56 e-8$ \\
& 5 & 0.00003105 & $2.86 e-13$ \\
\hline \multirow{3}{*}{$25+5 i$} & 1 & 0.00974880 & 0.00004491 \\
& 3 & $5.46 e-6$ & $2.66 e-10$ \\
& 5 & $3.67 e-9$ & $3.78 e-14$ \\
\hline \multirow{3}{*}{50} & 1 & 0.00498611 & 0.00001207 \\
& 3 & $6.85 e-7$ & $2.15 e-11$ \\
& 5 & $1.15 e-10$ & $7.93 e-15$ \\
\hline
\end{tabular}

\begin{tabular}{c|c|c|c|}
\hline \multicolumn{3}{|c}{$z=-1+i / 4$} \\
\hline \hline \multirow{3}{*}{0.75} & $n$ & Olver's method & Formula (31) \\
& 1 & 1.06271455 & 0.50808214 \\
& 3 & 0.87941096 & 0.01338941 \\
& 5 & 0.91915445 & 0.00005423 \\
\hline \multirow{5}{*}{5} & 1 & 0.21929092 & 0.02356432 \\
& 3 & 0.00507404 & 0.00013029 \\
& 5 & 0.00013229 & $4.81 e-7$ \\
\hline \multirow{5}{*}{25} & 1 & 0.03935288 & 0.00089998 \\
& 3 & 0.00002050 & $1.38 e-7$ \\
& 5 & $1.39 e-8$ & $8.51 e-12$ \\
\hline \multirow{3}{*}{50} & 1 & 0.01924853 & 0.00023144 \\
& 3 & $2.35 e-6$ & $9.05 e-9$ \\
& 5 & $3.85 e-10$ & $1.44 e-13$ \\
\hline
\end{tabular}

Table 2: Numerical experiments about the relative errors in the approximation of the solution of problem (29) using Olver's method and the iterative method (31) for different values of $\Lambda$ and $n$. For the given values of $n$, the relative errors correspond to the approximate solution $y_{n}^{-}(z)$ for the iterative method and the approximate solution $a_{n+1} Y_{n+1}^{+}(z)+b_{n+1} Y_{n+1}^{-}(z)$ for Olver's method.

first instance, $Y_{n}^{+}(z)$ and $Y_{n}^{-}(z)$ from (24) and (25); then, we must compute the coefficients $a_{n}, b_{n}$ and $c_{n}$ at every step $n$ of the approximation to obtain $y_{n}^{+}(z)$ and $y_{n}^{-}(z)$ as the linear combinations $y_{n}^{+}(z)=c_{n} Y_{n}^{+}(z)$ and $y_{n}^{-}(z)=a_{n} Y_{n}^{+}(z)+b_{n} Y_{n}^{-}(z)$.

We start the sequence $(15)$ at $y_{0}^{+}(z)=\bar{y}_{0}$, a function bounded at $z=0$. We observe in (15) that the iteration $y_{n}^{+} \rightarrow y_{n+1}^{+}$keeps this property, as all the terms of the sequence $y_{n}^{+}$are bounded at $z=0$. And the sequence converges to a function of the unique one-dimensional space of solutions of equation (6) that are bounded at $z=0$. The situation is different with the recurrence (16). Except for the above mentioned one-dimensional space, the whole twodimensional space of solutions of the equation (6) consists of functions unbounded at $z=0$. Then, even if we start the sequence $y_{n}^{-}(z)$ with a function $y_{0}^{-}(z)$ analytic at $z=0$, that is, if we take $\tilde{y}_{1}=0$ and $\tilde{y}_{0} \neq 0$ in (16), the iteration $y_{n}^{-} \rightarrow y_{n+1}^{-}$, in general, does not keep this property, it falls off the one dimensional space of bounded solutions at $z=0$.

The situation described in the above paragraph is one side of the coin. The other side is the fact that, for the equation (6), it is possible to get asymptotic approximations for the unique solution of an initial value problem with initial data prescribed at $z=0$ : problem (7), using either the fixed point technique or Olver's method. These methods do not work when we want to approximate a second solution independent of the previous one using an initial value problem with initial data prescribed at $z=0$ : observe that we cannot set $z_{0}=0$ in the recursion (16) as the integrals become meaningless. Something similar occurs in Olver's method: we cannot find a bound for the remainder $R_{n}^{-}(z)$ if we set $z_{0}=0$, as the kernel $1-(z / t)^{2 \Lambda-1}$ is not bounded for $t \in[0, z]$. That is why we have considered the initial value problem (8) with $z_{0} \neq 0$.

The error bounds (14) and (17) are not uniform in $z$. This means that the convergent and 
asymptotic character of the expansions of Section 3 for the unique solutions of the initial value problems (7) and (8) is proved only over bounded subsets of $\mathcal{D}$. On the other hand, when $A_{n}^{\prime}$ and $g$ are integrable in unbounded paths $\mathcal{L}$, the bound $(26)$ shows the uniform character of Olver's asymptotic expansions of Section 5 for two independent solutions of the differential equation $z y^{\prime \prime}+2 \Lambda y^{\prime}=g y$. The situation in Olver's theory in the cases $m \neq 2$ is slightly different: Olver obtains asymptotic expansions of two independent solutions of the differential equation $z^{m} u^{\prime \prime}-\tilde{\Lambda}^{2} u=z^{m} h(z) u$ in unbounded domains for $z$.

\section{Acknowledgments}

The Dirección General de Ciencia y Tecnología (REF. MTM2010-21037) is acknowledged by its financial support. The referees are acknowledged by their comments and suggestions that have contributed to improve the presentation of the paper.

\section{References}

[1] P. B. Bailey, L. F. Shampine And P. E. Waltman, Nonlinear Two Point Boundary Value Problems, Academic Press, New York, 1968.

[2] E. Coddington and N. Levinson, Theory of ordinary differential equations, McGraw-Hill, 1955.

[3] J. L. López, The Liouville-Neumann expansion at a regular singular point, J. Diff. Eq. Appl., 15 no. 2 (2009) 119-132.

[4] J. L. López, Olver's asymptotic method revisited. Case I, J. Math. Anal. Appl., 395 no. 2 (2012) 578-586.

[5] F. W. J. Olver, Asymptotics and Special Functions, Academic Press, New York, 1974.

[6] F. W. J. Olver, L. C. Maximon, Bessel functions, in: NIST Handbook of Mathematical Functions, Cambridge University Press, Cambridge, 2010, pp. 215-286 (Chapter 10). http://dlmf.nist.gov/10.

[7] I. Stackgold, Green's functions and Boundary Value Problems, John Wiley \& Sons, New York, 1998, Second Edition. 